\title{
Pancharatnam-Berry Optical Elements for Spin and Orbital Angular Momentum Division Demultiplexing
}

\author{
Gianluca Ruffato ${ }^{1,2}$, Pietro Capaldo ${ }^{3}$, Michele Massari ${ }^{2,3}$,, Alessia Mezzadrelli ${ }^{1,2}$ \\ and Filippo Romanato $1,2,3, *$ \\ 1 Department of Physics and Astronomy 'G. Galilei', University of Padova, via Marzolo 8, 35131 Padova, Italy; \\ gianluca.ruffato@unipd.it (G.R.); alessia.mezzadrelli@studenti.unipd.it (A.M.) \\ 2 Laboratory for Nanofabrication of Nanodevices (LaNN), EcamRicert, Corso Stati Uniti 4, \\ 35127 Padova, Italy; massari@iom.cnr.it \\ 3 CNR-INFM TASC IOM National Laboratory, S.S. 14 Km 163.5, Basovizza, 34012 Trieste, Italy; \\ capaldo@iom.cnr.it \\ * Correspondence: filippo.romanato@unipd.it
}

Received: 21 September 2018; Accepted: 31 October 2018; Published: 3 November 2018

\begin{abstract}
A Pancharatnam-Berry optical element is designed, fabricated, and optically characterized for the demultiplexing of beams with different polarization and orbital angular momentum states at the telecom wavelength of $1310 \mathrm{~nm}$. The geometric phase control is achieved by fabricating properly-oriented subwavelength gratings on a silicon substrate, inducing a spatially-variant form birefringence. The digital grating pattern is transferred to the silicon substrate with a two-step nanofabrication protocol, using inductively coupled plasma reactive ion etching to transfer the resist pattern generated with high-resolution electron beam lithography. The optical characterization of the sample confirms the expected capability to sort circularly polarized optical beams with different handedness and orbital angular momentum. Encompassing optical element design and silicon photonics, the designed silicon metasurface paves the way to innovative devices for total angular momentum mode division multiplexing with unprecedented levels of integration.
\end{abstract}

Keywords: orbital angular momentum; mode division multiplexing; polarization division multiplexing; Pancharatnam-Berry optical elements; metasurfaces; subwavelength gratings; electron beam lithography; reactive ion etching; silicon

\section{Introduction}

In order to face the worldwide ever-increasing demand for bandwidth, different methods have been developed and employed to exploit the several degrees of freedom of light for encoding information and increasing the capacity of optical networks. However, also the combination of well-established multiplexing techniques based on wavelength, polarization, time, amplitude, and phase, the modulation of light cannot exceed the physical limit of single-mode optical fibers. On the other hand, moving to a multi-mode transmission medium opens the network to the exploitation of the different orthogonal modes as distinct propagation channels, in the so-called mode division multiplexing (MDM) [1].

In the cylindrically symmetric framework of optical fibers, modes can be labeled on the basis of the carried orbital angular momentum (OAM) and present a well-defined circular polarization state, associated with the spin angular momentum (SAM) [2]. OAM modes are endowed with a peculiar helical wavefront $\exp (i \ell \varphi)(\ell=0, \pm 1, \pm 2, \ldots)$, with $\ell$ being the amount of OAM per photon in units of $h$, and exhibit an axially symmetric intensity distribution around a central dark point [3]. OAM-MDM 
has been demonstrated to promote an increase in information capacity for both quantum [4] and classical communications [5,6]. The multiplexing of angular momentum states of light has also been successfully applied to increase the set of transmission channels at the same wavelength in free space, where the number of supported OAM modes is theoretically unbounded [7].

A pivotal part of a communication system based on OAM-MDM is represented by the so-called multiplexer, i.e., the device located at the entry side of the optical link allowing the generation of a bunch of collimated and superimposed orthogonal beams. At the output end, the beam is sorted by the demultiplexer, which is usually constituted of a multiplexing element working in reverse. Several solutions have been designed and presented in the literature, exhibiting different levels of complexity, efficiency, and integration [8].

Industry requirements favor technological solutions that are efficient, suitable to miniaturization and integration, compatible with mass production, and potentially encompassing many multiplexing techniques. In the quest for miniaturization, we have recently demonstrated the design and fabrication of 3D multi-level diffractive optics [9-12] in the visible range performing OAM mode generation and demultiplexing, based either on OAM mode projection [13] or log-pol optical transformation [14]. With respect to bulky refractive optical elements, the diffractive counterpart offers a flat and integrable solution, especially when short focal lengths are required. However, their fabrication is optimized for a narrow bandwidth, and their efficiency drops when operating at a different wavelength. The optical thickness is proportional to the design wavelength, and inversely proportional to the refractive index of the material. If the transparency of silicon in the infrared telecom range suggests the usage of this high-refractive index material to further reduce the thickness, on the other hand, the realization of a 3D surface relief silicon pattern is undoubtedly challenging.

In this paper, an alternative implementation is shown and treated by realizing demultiplexing elements for OAM-MDM in the form of silicon Pancharatnam-Berry optical elements (PBOEs) for the telecom wavelength of $1310 \mathrm{~nm}$. Unlike diffractive and refractive elements, in PBOEs the phase is not introduced through optical path differences, but rather results from the geometrical phase that occurs with the space-variant polarization manipulation in the spatial domain [15]. Such optical elements are realized in the form of spatially variant phase plates, whose optical axis varies point-by-point in order to generate the desired phase profile. With respect to diffractive optics, they exhibit a broader operating band [16], since the wavefront is tailored by the geometry of the optics. Moreover, since their optical response is inherently dependent on the polarization state of the input beam [17], polarization-division multiplexing (PDM) can be included by properly engineering the optical element [18].

In this work, the birefringence of the optics subunit has been obtained artificially by structuring the substrate with a digital subwavelength grating. The originated metasurface is characterized by two local effective refractive indices, referring to the extraordinary and ordinary propagation axes, which are perpendicular and parallel to the local grating orientation, respectively [19]. By combining different high-resolution nanofabrication techniques, the PBOEs of spatially rotating subwavelength gratings have been etched on a silicon substrate, realizing a two-dimensional (2D) digital structure.

We considered the demultiplexing method based on OAM mode projection, and we designed and fabricated for the first time a silicon metasurface performing both OAM-MDM and PDM. The optical characterization of the sorter has been shown at the telecom wavelength of $1310 \mathrm{~nm}$, demonstrating the expected capability to separate modes with different spin and orbital angular momentum states with a single optical platform. 


\section{Materials and Methods}

\subsection{Phase Pattern Calculation}

The phase pattern of a diffractive optics intended for analyzing the OAM spectrum of the incident light field in the set $\left\{\ell_{j}\right\}$ is given by the linear combination over a group of $n$ orthogonal modes $\left\{\psi_{j}=R_{j}(\rho, \vartheta) \exp \left(i \ell_{j} \vartheta\right)\right\}$ as it follows [20]:

$$
\Omega(\rho, \vartheta)=\arg \left\{\sum_{j=1}^{n} c_{j} \psi_{j}^{*} \exp \left[i \rho \beta_{j} \cos \left(\vartheta-\vartheta_{j}\right)\right]\right\},
$$

where $\left\{\left(\beta_{j}, \vartheta_{j}\right)\right\}$ are the $n$ vectors of carriers spatial frequencies in polar coordinates, $c_{j}$ are complex coefficients whose modulus is given arbitrarily, usually unitary, and the phases are free parameters of the task, fitted in such a manner that Equation (1) becomes an exact equality. The coefficients are given by the following relation:

$$
c_{j}=\int_{0}^{2 \pi} d \vartheta \int_{0}^{+\infty} \psi_{j} \exp \left(i \Omega_{D O E}\right) \exp \left[-i \rho \beta_{j} \cos \left(\vartheta-\vartheta_{j}\right)\right] \rho d \rho,
$$

The positions $\left\{\left(r_{j}, \varphi_{j}\right)\right\}$ of the corresponding signal spots in the far-field are given by:

$$
\begin{gathered}
r_{j}=\beta_{j} \frac{f}{k} \\
\varphi_{j}=\vartheta_{j}
\end{gathered}
$$

with $f$ being the focal length of the lens exploited for far-field reconstruction in $f$ - $f$ configuration, and $k=2 \pi / \lambda$, where $\lambda$ is the wavelength. When an OAM beam illuminates the optical element, its projection over the total OAM set appears in the far-field, and a bright spot forms at the position corresponding to the carried OAM value (Figure 1) (see Appendix A for more details).

When the optical element is realized in a metasurface form with total polarization conversion ( $\pi$ retardation), the two orthogonal circular polarizations experience opposite phase patterns, as it follows (see Appendix B):

$$
T\left(\begin{array}{c}
1 \\
\pm i
\end{array}\right)=-i \exp ( \pm i \Omega)\left(\begin{array}{c}
1 \\
\mp i
\end{array}\right)
$$

with $T$ being the transmission matrix of the optical element, and $[1,+i]$ and $[1,-i]$ being the vectors of right-handed and left-handed circular polarizations, in Jones matrix formalism. Therefore, orthogonal polarizations experience two different phase patterns:

$$
\begin{aligned}
& \Omega^{(+)}(\rho, \vartheta)=\arg \left\{\sum_{j=1}^{n} c_{j} R_{j}^{*} \exp \left[-i \ell_{j} \vartheta+i \rho \beta_{j} \cos \left(\vartheta-\vartheta_{j}\right)\right]\right\} \\
& \Omega^{(-)}(\rho, \vartheta)=\arg \left\{\sum_{j=1}^{n} c_{j} R_{j} \exp \left[+i \ell_{j} \vartheta-i \rho \beta_{j} \cos \left(\vartheta-\vartheta_{j}\right)\right]\right\}
\end{aligned}
$$

and the corresponding signal spots are detected at the following positions in the far field:

$$
\begin{aligned}
& r^{( \pm)}\left(\ell_{j}\right)=\beta_{j} f / k \\
& \varphi^{(+)}\left(\ell_{j}\right)=\vartheta_{j} \\
& \varphi^{(-)}\left(\ell_{j}\right)=\varphi^{(+)}\left(-\ell_{j}\right)+\pi
\end{aligned}
$$

As a consequence of the polarization-dependent optical response, a beam carrying OAM equal to $\ell$ and left-handed circular polarization generates a bright spot in the far-field at a position that is symmetric to the spot produced by the right-handed circularly polarized beam with an opposite OAM. 
Hence, it is convenient to design a metasurface performing demultiplexing over a symmetric set of modes and carefully choose the spatial frequency carriers in order to avoid channels overlap.

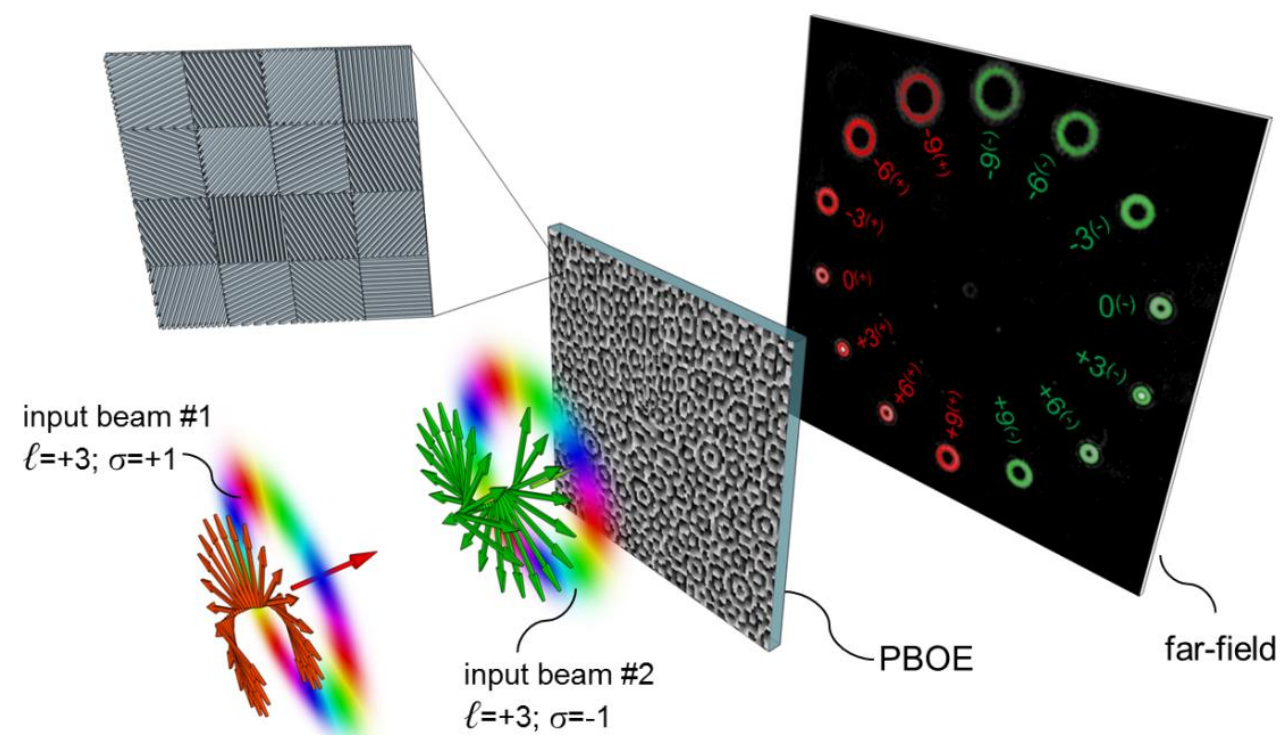

Figure 1. Scheme of the Pancharatnam-Berry optics working principle for an orbital angular momentum (OAM) beam sorting with the method of optical beam projection. When a circularly polarized OAM beam illuminates the optical element, a bright spot appears in the far field at a position depending on the carried OAM and on the polarization handedness. In the presented work, the Pancharatnam-Berry optical element (PBOE) is implemented in the form of a pixelated metasurface of rotated subwavelength grating.

A custom code implemented in MATLAB ${ }^{\circledR}$ is used to calculate the phase pattern for the desired set of OAM values $\left\{\ell_{j}\right\}$ and corresponding carrier spatial frequencies $\left\{\left(\beta_{j}, \vartheta_{j}\right)\right\}$. The implemented algorithm is based on a successive computation of the sum in Equation (1) and integrals in Equation (2), using the fast Fourier transform algorithm and considering definite limitations, as explained in Ruffato et al. [9], in particular the quantization of phase into 16 equally-spaced levels.

We limited our choice to OAM values in the set $\{-9,-6,-3,0,+3,+6,+9\}$ for a total of seven OAM channels (Figure 2a). We fixed the carrier spatial frequencies in such a way that the far-field peaks were arranged over a circle of constant radius $r$ at equally spaced angular positions (see Figure 2b), specified as follows:

$$
\begin{gathered}
r_{j}=r=\beta \frac{f}{k} \\
\varphi_{j}=\frac{\pi}{2}+j \frac{2 \pi}{14}
\end{gathered}
$$

where $j=1, \ldots, 7$. Therefore, the two orthogonal polarizations are sorted over the two hemicycles without overlapping, as shown in the scheme in Figure 2b. In fact, according to Equation (6), the far-field intensity peaks are expected to be at the following angular positions:

$$
\begin{aligned}
& \varphi^{(+)}\left(\ell_{j}\right)=\frac{\pi}{2}+j \frac{2 \pi}{14} \\
& \varphi^{(-)}\left(\ell_{j}\right)=\frac{\pi}{2}+(7-j+1) \frac{2 \pi}{14}+\pi=\frac{\pi}{2}-(j-1) \frac{2 \pi}{14}
\end{aligned} .
$$


(a)

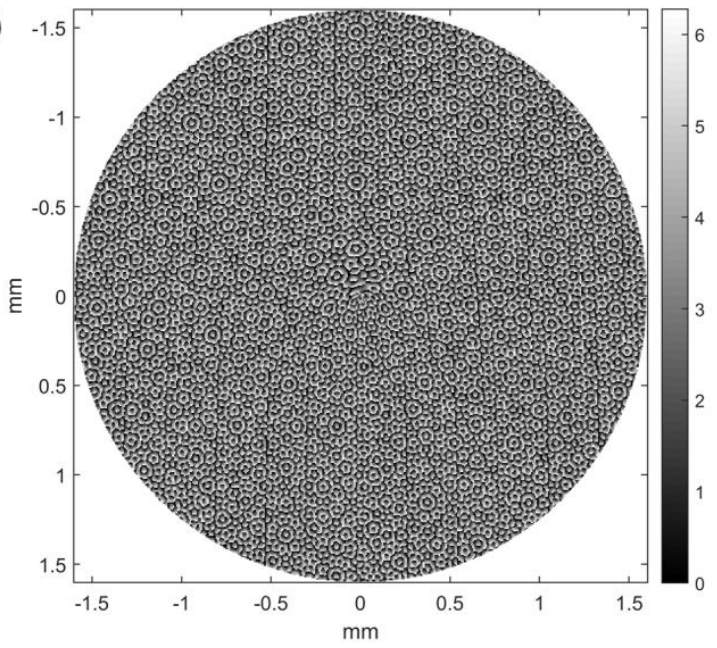

(b)

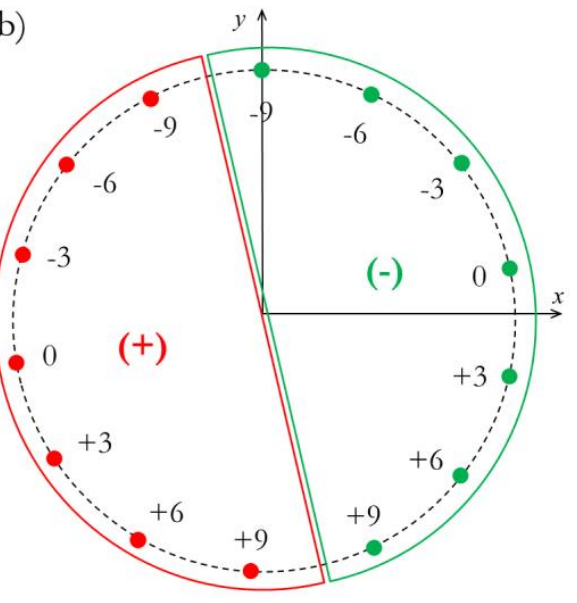

Figure 2. (a) Numerical phase pattern for the sorting of OAM beams in the range $\{-9,-6,-3,0,+3$, $+6,+9\}$, with 16 phase levels. Pixel size: $6.125 \mu \mathrm{m} \times 6.125 \mu \mathrm{m}$. Radius size: 256 pixels. (b) Far-field channel constellation for the given OAM set and circular polarization states.

\subsection{Subwavelength Grating Design}

The space-variant manipulation of polarization can be achieved with a both inhomogeneous and anisotropic medium, which is constituted of a birefringent material whose optical axis is not fixed. Such an element can be realized artificially in the form of subwavelength gratings, whose ridges' orientation changes pixel-by-pixel, inducing a spatially variant form birefringence [19]. Thus, the key element of the metasurface is represented by the subwavelength linear grating cell, whose local orientation $\theta(x, y)$ is chosen in order to transfer the desired geometric phase delay $\Omega(x, y)$ to the incident light, according to (see Appendix B):

$$
\theta(x, y)=\frac{\Omega(x, y)}{2}
$$

Digital subwavelength gratings have birefringent properties arising from the specific geometric structure that leads to a form birefringence where the grating acquires uniaxial crystal properties. Its optical response is characterized by two different refractive indices referring to the extraordinary and ordinary propagation axes, which are perpendicular and parallel to the grating lines, respectively. In order to achieve complete polarization conversion, the two refractive indices and the grating thickness should be properly engineered in order to get a phase delay equal to $\pi$ between TE and TM polarizations, i.e., linear polarizations perpendicular and parallel to the grating vector, respectively. Simulation sessions considering rigorous coupled-wave analysis (RCWA) [21,22] have been performed to find the best geometric parameters for a silicon digital grating working at the wavelength of $1310 \mathrm{~nm}$ at normal incidence. For a thickness of $535 \mathrm{~nm}$, the $\pi$-delay configurations of the duty cycle and grating period are reported in Figure 3. We selected a duty cycle of 0.5 , corresponding to a grating period of around $290 \mathrm{~nm}$. 


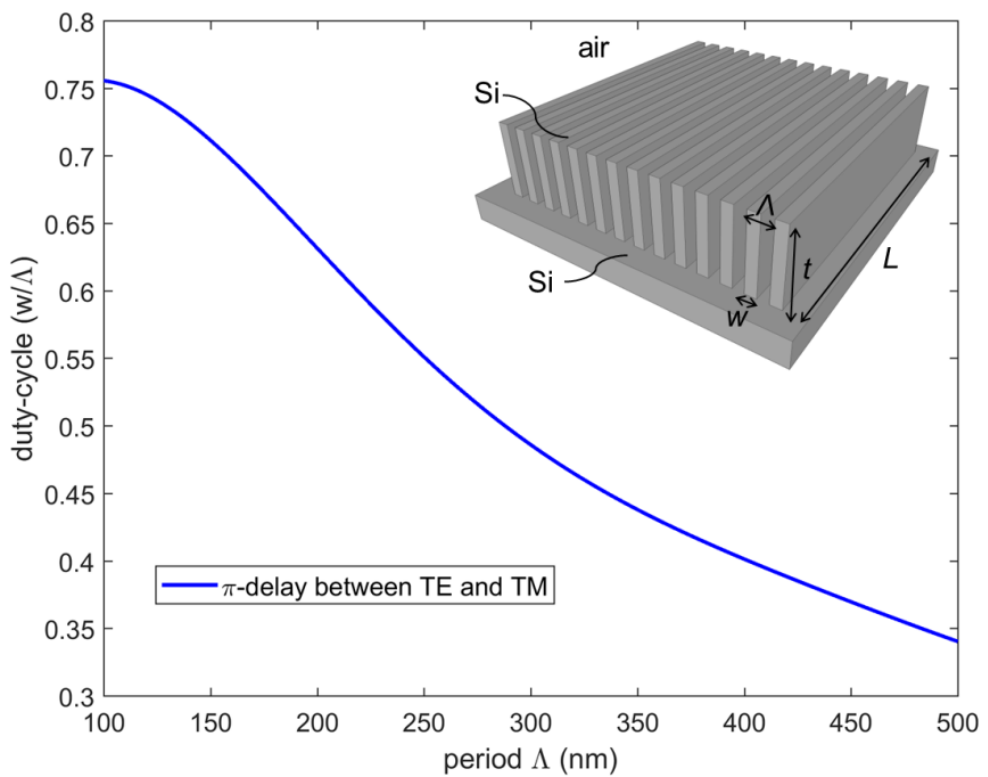

Figure 3. Configurations of duty cycle and period for a silicon subwavelength grating, providing a $\pi$-delay retardation between TE and TM polarizations, for a wavelength of $1310 \mathrm{~nm}$ at normal incidence. Numerical data obtained with rigorous coupled-wave analysis (RCWA) for a grating thickness of $535 \mathrm{~nm}$.

\subsection{Fabrication}

The fabrication protocol for pattern transfer onto silicon is divided into two distinct processes, in sequence: fabrication of the resist mask with electron beam lithography (EBL) of a resist film spun over the silicon substrate, and transfer of the mask profile thereon with inductively coupled plasma-reactive ion etching (ICP-RIE).

Electron beam lithography is the ideal technique for transfering the computational design from a digital stored format to a surface relief pattern with high-resolution profiles [23]. It is a maskless process: a high-powered focused electron beam draws the high-resolution pattern directly onto an electron-sensitive polymeric layer. The resist used in this work (AR-671.02, ALLRESIST GmbH, Strausberg, Germany) has a positive tone, so the interaction with the electron beam breaks the polymeric chain, making the polymer more soluble in its specific developer. A thin layer was spun at $2000 \mathrm{rpm}$, obtaining a thickness of around $200 \mathrm{~nm}$, followed by a hotplate baking process at $150{ }^{\circ} \mathrm{C}$ for three minutes. A JBX-6300FS EBL machine (JEOL, Tokyo, Japan), with $12 \mathrm{MHz}$ and 5-nm resolution, working at $100 \mathrm{kV}$ with a current of $100 \mathrm{pA}$ was used, selecting an exposition dose of $400 \mu \mathrm{C} / \mathrm{cm}^{2}$ from previous dose matrix analyses. After development in an isopropyl alcohol (IPA): deionized water 3:7 solution for $60 \mathrm{~s}$, the exposed area results to be removed.

Then, the generated pattern was used as a mask in ICP-RIE configuration. STS MESC MULTIPLEX ICP (SemiStar Corp, San Jose, CA, USA) has been used where the plasma is inductively coupled at 13.56 MHz via a matching unit and coil assembly. The ICP-RIE recipe that was used to transfer the resist pattern onto the silicon substrate is composed by three sub-steps: $\mathrm{O}_{2}$ stripping of the residual layer removal characterized by a $40-\mathrm{sccm} \mathrm{O}_{2}$ flow rate, $200 \mathrm{~W}$ of radio-frequency (RF) power of coil for the generation of plasma, 4 mTorr of chamber pressure, and an RF platen power of $10 \mathrm{~W}$ for the acceleration of the ions on top of the sample. The etching step is performed in a three-gases mix of plasma $\left(\mathrm{C}_{4} \mathrm{~F}_{8}\right.$ at a flow rate of $60 \mathrm{sccm}, \mathrm{SF}_{6}$ at $30 \mathrm{sccm}$, and Ar at $\left.10 \mathrm{sccm}\right)$ with a coil RF power of $400 \mathrm{~W}$, a RF platen power of $20 \mathrm{~W}$ (corresponding to an acceleration bias of $90 \mathrm{~V}$ ), and a chamber pressure of 8 mTorr. Finally, an $\mathrm{O}_{2}$ cleaning step was carried out, which was characterized by an $\mathrm{O}_{2}$ flow rate of $50 \mathrm{sccm}$, a coil RF power of $800 \mathrm{~W}$, an RF platen power of $20 \mathrm{~W}$, and a chamber pressure of 20 mTorr. In order to have pattern transfer from the EBL-lithographed resist to the Si substrate, 
a 7-s stripping process in $\mathrm{O}_{2}$ plasma has been performed, and successively, an etching time of $1 \mathrm{~min}$ and $12 \mathrm{~s}$ in fluorine-based plasma occurred.

A thermal nanoimprint lithography (T-NIL) process was conducted using a Paul Otto Weber hydraulic press with heating/cooling plates. This simple technique enables replicating patterns with high resolution [24,25]. A mold with nanostructures on its surface was used to deform a thin resist deposited on a substrate that has to be heated above its glass transition temperature. At the end, the resist is cooled below its glass transition temperature before being separated from the mold. A first T-NIL process was performed using the previous etched EBL master as cast after a silanization process with Trichloro(1H,1H,2H,2H-perfluorooctyl)silane PFOTS (Thermo Fisher (Kandel) GmbH, Karlsruhe, Germany) [26,27]. MR-I 7010E was spun on a silicon wafer at $1750 \mathrm{rpm}$ to obtain a thickness of about $116 \mathrm{~nm}$, and a soft bake was conducted for $2 \mathrm{~min}$ at $140{ }^{\circ} \mathrm{C}$ on the hot plate. The sample was placed in contact with the master within a system of compliances in order to homogenize the temperature and pressure on the entire surface. The T-NIL process was carried out at $100{ }^{\circ} \mathrm{C}$ for an imprinting time of $10 \mathrm{~min}$ at a pressure of 100 bar. Once the imprinting time was over, the heating was turned off, and maintaining the pressure at $100 \mathrm{bar}$, a temperature decreases until $35^{\circ} \mathrm{C}$ occurred.

After an $\mathrm{O}_{2}$ treatment was performed for $13 \mathrm{~s}$ to remove the residual layer, a 10-nm $\mathrm{Cr}$ hard mask layer was deposited by e-gun evaporation, and the pattern transferring from the resist structure into the $\mathrm{Cr}$ mask was carried out by lift-off in a sonicated acetone bath for $3 \mathrm{~min}$.

A final ICP-RIE process was then performed: an $\mathrm{O}_{2}$ treatment was considered for $30 \mathrm{~s}$ to remove the last resist contaminants; then, pattern transfer in the fluorine-based plasma for 3 min and $9 \mathrm{~s}$ occurred. The etching time was finely tuned in order to obtain a final depth of around $535 \mathrm{~nm}$, as prescribed by the numerical results in Figure 3 for a grating period of $290 \mathrm{~nm}$ and a duty cycle of around 0.5. In Figure 4, inspections of the final sample via scanning electron microscopy (SEM) are reported. In particular, the well-defined line profile can be appreciated as proof of the success of the fabrication recipe for pattern transfer onto a silicon substrate.

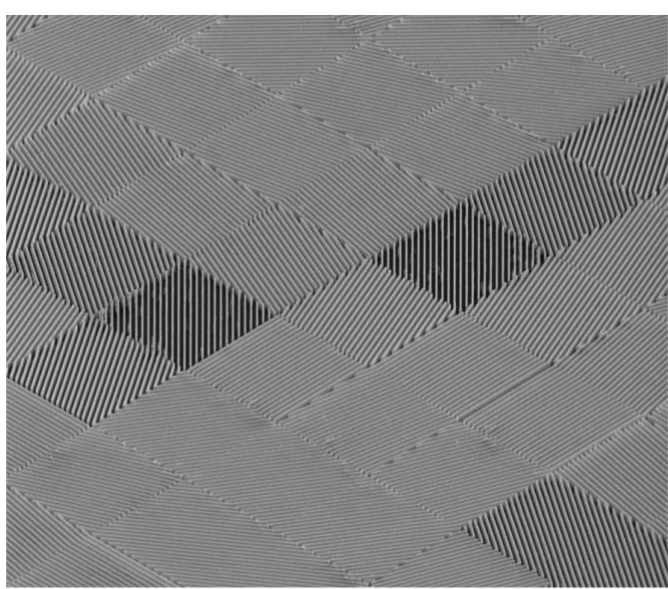

$5 \mu \mathrm{m}$

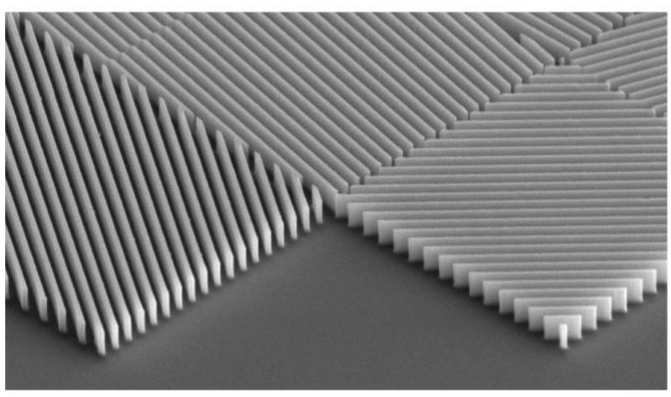

$2 \mu \mathrm{m}$

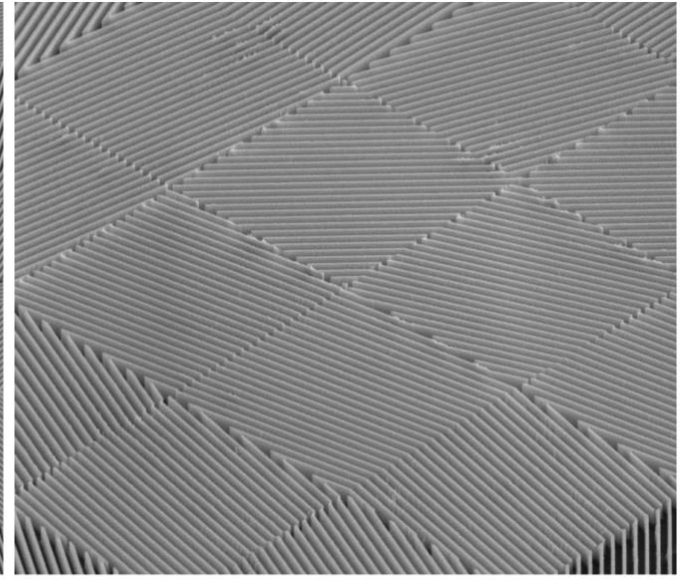

$2.5 \mu \mathrm{m}$

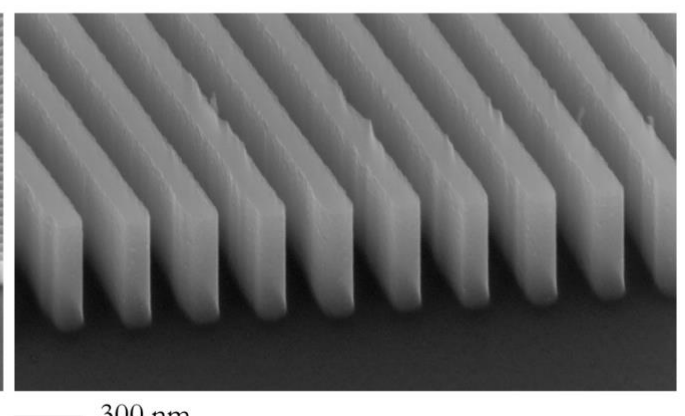

$300 \mathrm{~nm}$

Figure 4. SEM inspections of the fabricated PBOE on a silicon substrate. Grating period $\Lambda=290 \mathrm{~nm}$, duty cycle 0.5 , thickness: $535 \mathrm{~nm}$, pixel size: $6.125 \mu \mathrm{m}, 16$ rotation angles. 


\subsection{Optical Characterization Setup}

The optical behavior of the sample has been tested for illumination under beams carrying orbital angular momentum, which were generated with an Liquid-Crystal on Silicon (LCoS) spatial light modulator (SLM) (pixel pitch $12.5 \mu \mathrm{m}$, X13267-08, Hamamatsu, Shizuoka, Japan) using a phase and amplitude modulation technique [28]. The experimental setup is depicted in Figure 5. The output of a DFB laser $(\lambda=1310 \mathrm{~nm})$ is collimated at the end of the single mode fiber with an aspheric lens with a focal length $f_{\mathrm{F}}=7.5 \mathrm{~mm}$ (A375TM-C, Thorlabs, Newton, NJ, USA), which is linearly polarized and expanded with a first telescope $\left(f_{1}=3.5 \mathrm{~cm}, f_{2}=10.0 \mathrm{~cm}\right.$, Thorlabs, NJ, USA) before illuminating the display of the SLM. A 50:50 beam splitter (Thorlabs, Newton, NJ, USA) is inserted after the telescope in order to retain a coherent Gaussian beam for interferometric analysis. Then, a $4-f$ system $\left(f_{3}=20.0 \mathrm{~cm}\right.$, $f_{4}=12.5 \mathrm{~cm}$, Thorlabs, Newton, NJ, USA) with an aperture in the Fourier plane isolates and images the first-order encoded mode. A second 50:50 beam splitter is used to split the beam and check the input beam profile with a first camera (pixel pitch $15 \mu \mathrm{m}$, WiDy SWIR 640U-S, NIT, Verrières-le-Buisson, France). Afterwards, the OAM beam illuminates the patterned zone of the silicon sample, which is mounted on an XY translator with micrometer drives (ST1XY-S/M, Thorlabs, Newton, NJ, USA). The far field is collected by a second camera (WiDy SWIR 640U-S, NIT, Verrières-le-Buisson, France) that is placed at the back focal plane of a lens with $f_{5}=7.5 \mathrm{~cm}$ (Thorlabs, NJ, USA). A sequence of a linear polarizer (LPIREA100-C, Thorlabs, Newton, NJ, USA) and quarter-wave plate (WPQ10M-1310, Thorlabs, Newton, NJ, USA) is placed before and after the optical element, in reverse order, in order to generate and filter the desired circular polarization state. A Mach-Zehnder interferometric setup is added in order to analyze the phase pattern of the modes generated with the SLM.

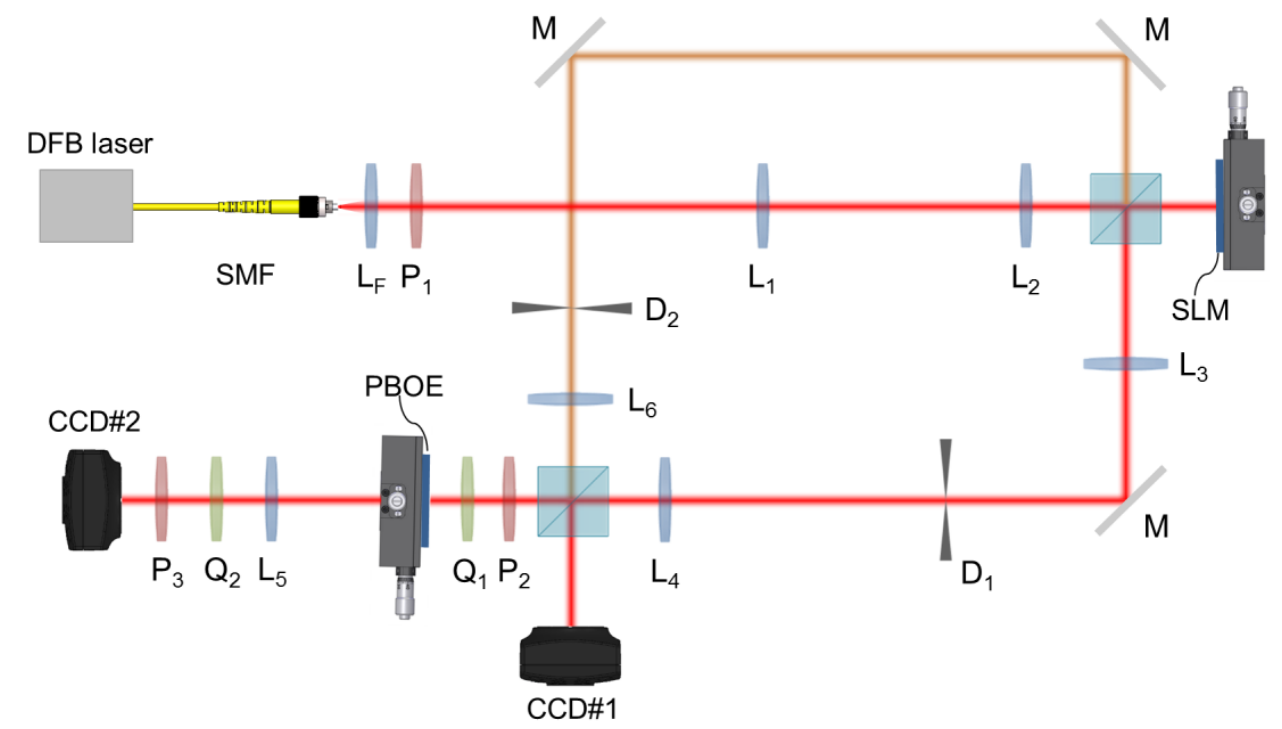

Figure 5. Scheme of the experimental setup used for the optical characterization of the fabricated sorter. The DFB laser output $(\lambda=1310 \mathrm{~nm})$ is collimated at the end of the single mode fiber by means of an aspheric lens with a focal length $f_{\mathrm{F}}=7.5 \mathrm{~mm}$, is linearly polarized $\left(\mathrm{P}_{1}\right)$ and is expanded $\left(f_{1}=3.5 \mathrm{~cm}\right.$, $\left.f_{2}=10.0 \mathrm{~cm}\right)$. The SLM first order is filtered $\left(\mathrm{D}_{1}\right)$ and resized $\left(f_{3}=20.0 \mathrm{~cm}, f_{4}=12.5 \mathrm{~cm}\right)$ before illuminating the sorter. A beam splitter (BS) is used both to check the input beam and collect the sorter output at the back focal plane of a fifth Fourier lens $\left(f_{5}=7.5 \mathrm{~cm}\right)$. A sequence of linear polarizers and quarter-wave plates is placed before $\left(\mathrm{P}_{2}, \mathrm{Q}_{1}\right)$ and after $\left(\mathrm{Q}_{2}, \mathrm{P}_{3}\right)$ in the optical element, in reverse order, in order to generate and filter the desired circular polarization states. A Mach-Zehnder interferometric setup is added in order to analyze the singularity order of the generated OAM beams. 


\section{Results}

The far field of the fabricated PBOE has been collected for input optical beams with well-defined orbital angular momentum and circular polarization states. Beams carrying OAM in the sorting set $\{-9,-6,-3,0,+3,+6,+9\}$ have been generated, in sequence, and circularly polarized before illuminating the sorter. The desired helical wavefront was loaded on a doughnut intensity profile and properly resized in order to fit the size of the optical element. Using a Mach-Zehnder interferometric branch, the interference pattern of the generated OAM beam was collected. Since the phase structure of an OAM beam presents $\ell$ intertwined helical phase fronts, with $\ell$ being the amount of OAM, the interference with a coaxial Gaussian beam generates a fringe pattern of $\ell$ spirals, whose helicity is given by the sign of $\ell$ [29]. As Figure 6a shows, the number and the helicity of the spiral arms in the interferograms denote the helical structure of the phase front and the carried OAM value and sign, as expected.

When an OAM beam illuminates the sorter, the far field is the result of the projection of the field over the mode set for which the element has been designed (see Appendix A for more details). Therefore, a bright spot appears in correspondence with the detected OAM value, when it is present, at the position given by the channel constellation in Figure $2 b$. In Figure $6 b, c$, the far field is reported for input OAM beams with right-handed and left-handed circular polarizations, respectively. As expected, the sorter separates the orthogonal polarization states onto two distinct hemicycles, and the OAM beams are correctly detected according to the scheme in Figure 2b. In Figure 7, normalized intensities are reported, exhibiting a well-defined diagonal channel response.

(a)
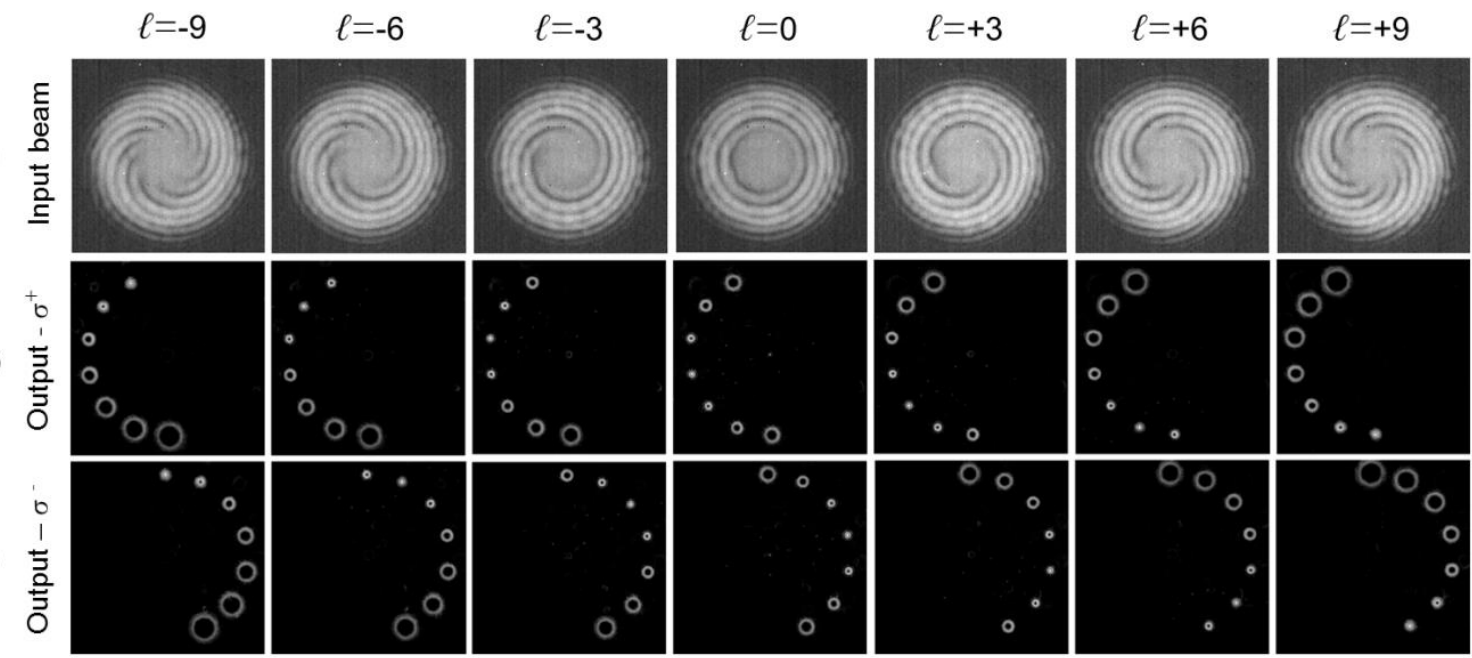

Figure 6. (a) Interference patterns of the OAM beams exploited for the sorter characterization. The number and twist handedness of the spirals is indicative of the carried OAM. Output of the fabricated OAM for input right-handed (b) and left-handed (c) circular polarization states. As expected, the far-field bright spot position is in accordance with the far-field channel constellation in Figure $2 \mathrm{~b}$. 


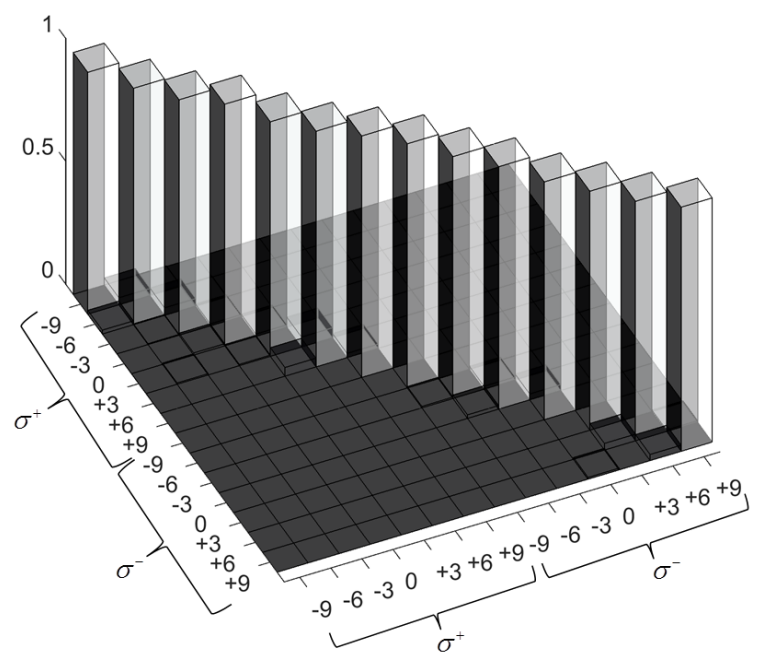

Figure 7. Total intensities in all of the detector regions for OAM mode input in the range $\{-9,-6,-3$, $0,+3,+6,+9\}$ and circular polarization of the experimental data. For each channel, the detection regions have the same size and are chosen to cover the central intensity peak area. Intensities are normalized to the total collected energy. The polarization labels $\sigma^{ \pm}$refers to the polarization state of the input channel (the output is cross-polarized).

\section{Discussion}

We presented the design, fabrication, and optical characterization of a silicon metasurface for the demultiplexing of optical beams with well-defined spin and orbital angular momentum states. We considered the demultiplexing method based on OAM mode projections, and we implemented the optical device in the form of a Pancharatnam-Berry optical element (PBOE). With respect to diffractive optics, the phase term is not originated by locally changing the thickness of the medium; instead, it arises from a local manipulation of the polarization due to an artificially-induced birefringence. In the presented study, the local anisotropy has been controlled by patterning properly oriented subwavelength gratings. The grating parameters have been selected after numerical simulations with RCWA in order to reproduce the behavior of a half-wave plate whose fast-axis orientation is equal to half the value of the sorter phase pattern at each point. The optical characterization with circularly polarized OAM beams confirms the expected capability of the metasurface to sort, at the same time, polarization and orbital angular momentum, therefore redoubling the number of channels of the diffractive counterpart. Moreover, the possibility to use silicon represents an important milestone. As a matter of fact, silicon has emerged as a popular material choice, not only for its high refractive index and very low absorption in the telecom spectral range, but also for its paramount technological relevance, since it represents the backbone of modern technology. With respect to plasmonic metamaterials, the relevance of silicon for optical elements is based not only on its optical material properties, low cost, and mature nanofabrication techniques, but also on the unique prospects that silicon nanostructures offer in terms of complementary metal oxide semiconductor (CMOS) compatibility and for integration into existing photonic architectures [30,31]. By encompassing silicon photonics and lens design, silicon metasurfaces pave the way to innovative optical devices performing OAM mode division multiplexing with a high level of integration and backward compatibility with complementary multiplexing techniques [18].

Author Contributions: Conceptualization, G.R. and F.R.; formal analysis, G.R.; investigation, G.R., M.M., P.C. and A.M.; software, G.R.; methodology, M.M., P.C. and A.M.; writing_original draft preparation, G.R., P.C. and M.M.; writing — review and editing, F.R.; supervision, F.R.; funding acquisition, F.R.

Funding: This work was supported by SM Optics S.r.l.-SIAE Group and by CEPOLISPE project 'VORTEX 2'.

Acknowledgments: The authors gratefully thank Giuseppe Parisi and Ing. Mauro Zontini for the interesting discussions during this work. 
Conflicts of Interest: The authors declare no conflict of interest.

\section{Appendix A}

We discuss more in details the far-field generation by a beam illuminating the sorter with phase function as in Equation (1). In particular, we consider an impinging perfect vortex with radius $R$ and ring width $\Delta R$, whose field can be described as follows [32]:

$$
U_{\ell}^{(i)}(\rho, \vartheta)=c \mathrm{e}^{i \ell \vartheta} e^{-\frac{(\rho-R)^{2}}{\Delta R^{2}}}
$$

$\ell$ being the carried OAM value and $c$ a normalization factor. In the paraxial approximation, the field at the back focal plane of a lens in $f-f$ configuration is given by [33]:

$$
U(r, \varphi)=\frac{e^{2 i k f}}{i \lambda f} \int_{0}^{+\infty} \rho d \rho \int_{0}^{2 \pi} U_{\ell}^{(i)}(\rho, \vartheta) e^{i \Omega(\rho, \vartheta)} e^{-i \frac{k \rho r}{f} \cos (\vartheta-\varphi)} d \vartheta
$$

$\lambda$ being the wavelength, $f$ the focal length, $\Omega$ the phase function of the sorter. As described above, the OAM sorter based on OAM projection over a set of $n$ OAM values can be described as the superposition of $n$ fork holograms with topological charges in the set $\{\ell j\}$ and spatial frequencies $\left\{\left(\beta_{j}, \vartheta_{j}\right)\right\}$ (see Equations (1) and (5)). Therefore, after substituting the definition (A1) and the sorter phase function, Equation (A2) becomes:

$$
U(r, \varphi)=\sum_{j=1}^{n} a_{j} \int_{0}^{+\infty} \rho d \rho \int_{0}^{2 \pi} e^{-\frac{(\rho-R)^{2}}{\Delta R^{2}}} e^{i\left(\ell-\ell_{j}\right) \vartheta} e^{i \rho \beta_{j} \cos \left(\vartheta-\vartheta_{j}\right)-i \frac{k \rho r}{f} \cos (\vartheta-\varphi)} d \vartheta,
$$

where constant parameters and normalization factors have been included in the coefficients $\left\{a_{j}\right\}$. After performing, inside each integral of the summation, the coordinate change: $\boldsymbol{r}_{j}^{\prime}=\boldsymbol{r}-f / k \boldsymbol{\beta}_{j}$, we get:

$$
U(r, \varphi)=\sum_{j=1}^{n} a_{j} \int_{0}^{+\infty} \rho d \rho \int_{0}^{2 \pi} e^{-\frac{(\rho-R)^{2}}{\Delta R^{2}}} e^{i\left(\ell-\ell_{j}\right) \vartheta} e^{-i \frac{k \rho r^{\prime} j}{f} \cos \left(\vartheta-\varphi^{\prime}{ }_{j}\right)} d \vartheta,
$$

Remembering the integral definition of Bessel functions of the first kind [34]:

$$
J_{m}(x)=\frac{1}{2 \pi i^{m}} \int_{0}^{2 \pi} d \vartheta e^{i m \vartheta} e^{-i m x \cos \vartheta}
$$

Equation (A4) becomes:

$$
U(r, \varphi)=\sum_{j=1}^{n} a_{j}^{\prime} e^{i\left(\ell-\ell_{j}\right) \varphi^{\prime}}{ }_{j}^{+\infty} \rho d \rho e^{-\frac{(\rho-R)^{2}}{\Delta R^{2}}} J_{\ell-\ell_{j}}\left(\frac{k \rho r^{\prime}}{f}\right)
$$

Under the approximation $\exp (x) \sim I_{m}(x)$, being $I_{n}$ the modified Bessel functions of the first kind, and applying the relation [34]:

$$
\int_{0}^{+\infty} x e^{-\alpha x^{2}} I_{m}(b x) J_{m}(c x) d x=\frac{1}{2 \alpha} \exp \left(\frac{b^{2}-c^{2}}{4 \alpha}\right) J_{m}\left(\frac{b c}{2 \alpha}\right),
$$


we finally obtain:

$$
U(r, \varphi)-\sum_{j=1}^{n} a_{j}^{\prime} e^{i\left(\ell-\ell_{j}\right) \varphi_{j}^{\prime}} \exp \left(-\frac{\Delta R^{2} k^{2} r^{\prime 2}}{4 f^{2}}\right) J_{\ell-\ell_{j}}\left(\frac{k R r_{j}^{\prime}}{f}\right)
$$

As described by the last equation, an input perfect vortex with OAM equal to $\ell$ is transformed into a set of $n$ Bessel-Gaussian beams with centers at the positions $\left\{\left(r_{j}, \varphi_{j}\right)\right\}=\left\{\left(f / k \beta_{j}, \vartheta_{j}\right)\right\}$ and azimuthal indices in the shifted set $\left\{\left(\ell-\ell_{j}\right)\right\}$. Only for $m=0$ the Bessel function of the first kind $J_{m}$ is endowed with a central peak, otherwise the intensity profile presents a central null surrounded by a first ring whose radius increases with the azimuthal index $m$. Therefore, as shown in Figure 6 and in Figure A1, for an input vortex with charge $\ell$ a bright spot appears only when a zero-order Bessel-Gaussian beam is generated, i.e., for $\ell_{j}=\ell$, while annular beams with different radii appear at the other positions. If the input OAM beam carries an OAM value which is not included in the sorting set of the designed sorter, e.g., $\ell=+2$, there is no possibility to obtain a zero-order Bessel-Gaussian beam, therefore no bright peak appears (see Figure A1). As a matter of fact, for the three cases depicted in figure, we get:

- input: $\ell=0$-> output: $\{0+9,0+6,0+3,0+0,0-3,0-6,0-9\}=\{+9,+6,+3,0,-3,-6,-9\}$

- input: $\ell=+3$-> output: $\{+3+9,+3+6,+3+3,+3+0,+3-3,+3-6,+3-9\}=\{+12,+9,+6,+3,0$, $-3,-6\}$

- input: $\ell=+2$-> output: $\{+2+9,+2+6,+2+3,+2+0,+2-3,+2-6,+2-9\}=\{+11,+8,+5,+2$, $-1,-4,-7\}$.

Analogous considerations are valid when considering other families of input OAM beams, for instance Laguerre-Gaussian beams.
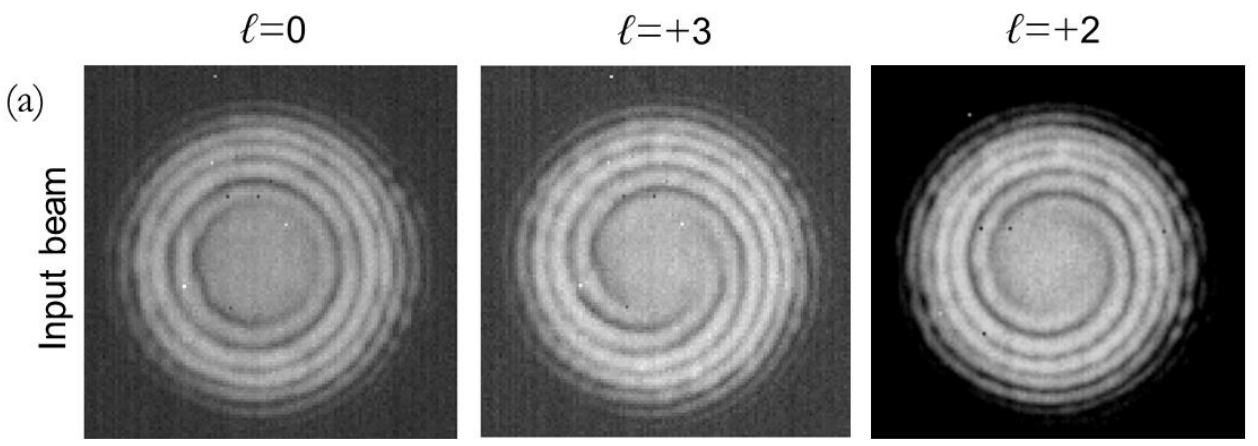

(b)
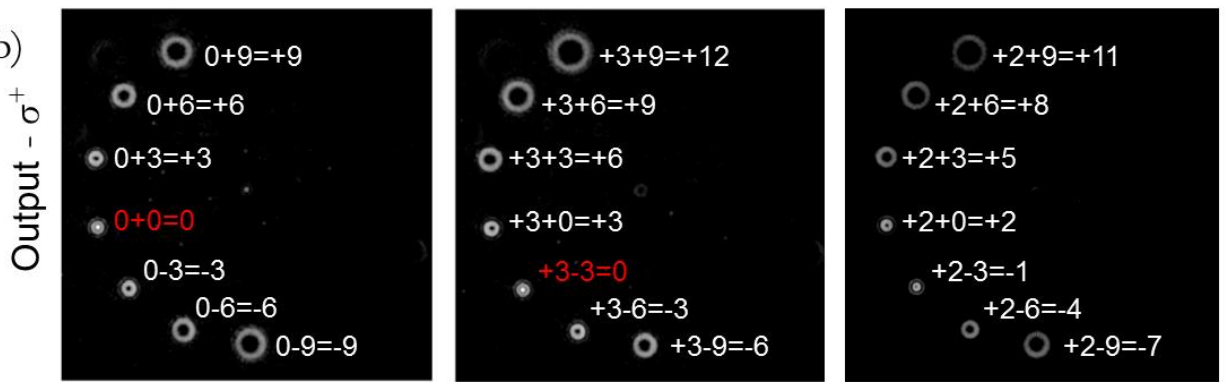

Figure A1. (a) Interference patterns of the OAM beams exploited for the sorter characterization, for $\ell=0,+2,+3$. The number and twist-handedness of the spirals is indicative of the carried OAM. (b) Output of the fabricated OAM for input right-handed circular polarization states. A bright spot appears when the azimuthal index of the generated far-field optical vortex is equal to zero. If the input beam carries an OAM value which is not included in the sorter set, as in the case of $\ell=+2$, no peak appears in far-field. 


\section{Appendix B}

For the benefit of the reader, we briefly summarize the optical behavior of a Pancharatnam-Berry optical element (PBOE) performing complete polarization conversion. It is convenient to describe the PBOE optical behavior with the use of Jones formalism, where the Transmission function $T$ of a subwavelength grating can be expressed, in the $(x, y)$ representation basis, by the matrix:

$$
T(x, y)=R[\theta(x, y)] \cdot \tau(\delta) R[\theta(x, y)]^{-1},
$$

where $\tau$ is the transmission matrix for a wave plate with phase retardation $\delta$, and $R$ the rotation matrix at each pixel, being $\theta(x, y)$ the local orientation of the sub-wavelength grating with respect to the $x$-axis. Their explicit form is given by:

$$
\begin{gathered}
R[\theta(x, y)]=\left[\begin{array}{cc}
\cos \theta(x, y) & -\sin \theta(x, y) \\
\sin \theta(x, y) & \cos \theta(x, y)
\end{array}\right], \\
\tau(\delta)=\left[\begin{array}{cc}
\exp (-i \delta / 2) & 0 \\
0 & \exp (i \delta / 2)
\end{array}\right],
\end{gathered}
$$

The phase retardation $\delta$ is determined by the geometry of the sub-wavelength grating in terms of period, duty-cycle and depth, and by the refractive index of the substrate material, and it is assumed to be constant for each pixel. For right and left circularly-polarized incident light, with corresponding Jones vectors $[1,+i]$ and $[1,-i]$ (the normalization factor $1 / \sqrt{2}$ has been omitted), the action of the matrix $T$ is given by:

$$
T(x, y)\left(\begin{array}{c}
1 \\
\pm i
\end{array}\right)=\cos \left(\frac{\delta}{2}\right)\left(\begin{array}{c}
1 \\
\pm i
\end{array}\right)-i \sin \left(\frac{\delta}{2}\right) e^{ \pm 2 i \theta(x, y)}\left(\begin{array}{c}
1 \\
\mp i
\end{array}\right)
$$

The resulting wave consists of two components: the zero order and the diffracted one. The zero order has the same polarization as the original wave and does not undergo any phase modification. On the other hand, the diffracted order has polarization orthogonal to that of the incoming wave, and its phase at each point is equal to twice the local orientation of the wave plate. It is worth noting how the cosine term, i.e., the zero-order term, can be eliminated by designing the grating parameters in such a way that the retardation phase equals $\pi$. In this case, the metasurface provides pure phase modulation, with the obtained phase being twice the rotation angle. This dependence is geometrical in nature and originates solely from local changes in the polarization state of the emerging beam:

$$
T(x, y)\left(\begin{array}{c}
1 \\
\pm i
\end{array}\right)=-i e^{ \pm 2 i \theta(x, y)}\left(\begin{array}{c}
1 \\
\mp i
\end{array}\right),
$$

Therefore, the desired phase modulation can be achieved just by varying the orientation of the subwavelength structure of each pixel, and a potentially continuous phase modulation can be achieved using a binary grating, eliminating the need for complicated multiple step and grey scale fabrication procedures.

\section{References}

1. Agrell, E.; Karlsson, M.; Chraplyvy, A.R.; Richardson, D.J.; Krummrich, P.M.; Winzer, P.; Roberts, K.; Fisher, J.K.; Savory, S.J.; Eggleton, B.J.; et al. Roadmap of optical communications. J. Opt. 2016, 18. [CrossRef]

2. Ramachandran, S.; Kristensen, P. Optical vortices in fiber. Nanophotonics 2013, 2, 455-474. [CrossRef]

3. Andrews, D.; Babiker, M. The Angular Momentum of Light; Cambridge University Press: Cambridge, UK, 2013; ISBN 9781107006348. 
4. Mirhosseini, M.; Magana-Loaiza, O.S.; O'Sullivan, M.N.; Rudenburg, B.; Malik, M.; Lavery, M.P.J.; Padgett, M.J.; Gauthier, D.J.; Boyd, R.W. High-dimensional quantum cryptography with twisted light. New J. Phys. 2015, 17. [CrossRef]

5. Bozinovic, N.; Yue, Y.; Ren, Y.; Tur, N.; Kristensen, P.; Huang, H.; Willner, A.E.; Ramachandran, S. Terabit-scale orbital angular momentum mode division multiplexing in fibers. Science 2013, 340, 1545-1548. [CrossRef] [PubMed]

6. Yu, S. Potential and challenges of using orbital angular momentum communications in optical interconnects. Opt. Express 2015, 23, 3075-3087. [CrossRef] [PubMed]

7. Willner, A.E.; Ren, Y.; Xie, G.; Yan, Y.; Li, L.; Zhao, Z.; Wang, J.; Tur, M.; Molish, A.F.; Ashrafi, S. Recent advances in high-capacity free-space optical and radio-frequency communications using orbital angular momentum multiplexing. Philos. Trans. A Math. Phys. Eng. Sci. 2017, 375. [CrossRef] [PubMed]

8. Wan, C.; Rui, G.; Chen, J.; Zhan, Q. Detection of photonic orbital angular momentum with micro- and nano-optical structures. Front. Optoelectron. 2017, 1-9. [CrossRef]

9. Ruffato, G.; Massari, M.; Romanato, F. Diffractive optics for combined spatial- and mode-division demultiplexing of optical vortices: Design, fabrication and optical characterization. Sci. Rep. 2016, 6, 24760. [CrossRef] [PubMed]

10. Ruffato, G.; Massari, M.; Romanato, F. Compact sorting of optical vortices by means of diffractive transformation optics. Opt. Lett. 2017, 42, 551-554. [CrossRef] [PubMed]

11. Ruffato, G.; Massari, M.; Parisi, G.; Romanato, F. Test of mode-division multiplexing and demultiplexing in free-space with diffractive transformation optics. Opt. Express 2017, 25, 7859-7868. [CrossRef] [PubMed]

12. Ruffato, G.; Girardi, M.; Massari, M.; Mafakheri, E.; Sephton, B.; Capaldo, P.; Forbes, A.; Romanato, F. A compact diffractive sorter for high-resolution demultiplexing of orbital angular momentum beams. Sci. Rep. 2018, 8. [CrossRef] [PubMed]

13. Gibson, G.; Courtial, J.; Padgett, M.J.; Vasnetsov, M.; Pas'ko, V.; Barnett, S.M.; Franke-Arnold, S. Free-space information transfer using light beams carrying orbital angular momentum. Opt. Express 2004, 12, 5448-5456. [CrossRef] [PubMed]

14. Berkhout, G.C.G.; Lavery, M.P.J.; Courtial, J.; Beijersbergen, M.W.; Padgett, M.J. Efficient sorting of orbital angular momentum states of light. Phys. Rev. Lett. 2010, 105. [CrossRef] [PubMed]

15. Roux, F.S. Geometric phase lens. J. Opt. Soc. Am. A 2006, 23, 476-482. [CrossRef]

16. Chen, M.L.N.; Jiang, L.J.; Sha, W.E.I. Orbital Angular Momentum Generation and Detection by Geometric-Phase Based Metasurfaces. Appl. Sci. 2018, 8. [CrossRef]

17. Desiatov, B.; Mazurski, N.; Fainman, Y.; Levy, U. Polarization selective beam shaping using nanoscale dielectric metasurfaces. Opt. Express 2015, 23, 22611-22618. [CrossRef] [PubMed]

18. Li, Y.; Li, X.; Chen, L.; Pu, U.; Jin, J.; Hong, M.; Luo, X. Orbital Angular Momentum Multiplexing and Demultiplexing by a Single Metasurface. Adv. Opt. Mater. 2017, 5. [CrossRef]

19. Emoto, A.; Nishi, M.; Okada, M.; Manabe, S.; Matsui, S.; Kawatsuki, N.; Ono, H. Form birefringence in intrinsic birefringent media possessing a subwavelength structure. App. Opt. 2010, 49, 4355-4361. [CrossRef] [PubMed]

20. Kotlyar, V.V.; Khonina, S.N.; Soifer, V.A. Light field decomposition in angular harmonics by means of diffractive optics. J. Mod. Opt. 1998, 45, 1495-1506. [CrossRef]

21. Moharam, M.G.; Pommet, D.A.; Grann, E.B.; Gaylord, T.K. Stable implementation of the rigorous coupled-wave analysis for surface-relief gratings: Enhanced transmittance matrix approach. J. Opt. Soc. Am. A 1995, 12, 1077-1086. [CrossRef]

22. Kikuta, H.; Ohira, Y.; Kubo, H.; Iwata, K. Effective medium theory of two-dimensional subwavelength gratings in the non-quasi-static limit. J. Opt. Soc. Am. A 1998, 15, 1577-1585. [CrossRef]

23. Ruffato, G.; Rossi, R.; Massari, M.; Mafakheri, E.; Capaldo, P.; Romanato, F. Design, fabrication and characterization of Computer-Generated Holograms for anti-counterfeiting applications using OAM beams as light decoders. Sci. Rep. 2017, 7. [CrossRef] [PubMed]

24. Beck, M.; Graczyk, M.; Maximov, I.; Sarwe, E.L.; Ling, T.G.I.; Keil, M.; Montelius, L. Improving stamps for $10 \mathrm{~nm}$ level wafer scale nanoimprint lithography. Microelectron. Eng. 2002, 61-62, 441-448. [CrossRef]

25. Pozzato, A.; Grenci, G.; Birarda, G.; Tormen, M. Evaluation of a novolak based positive tone photoresist as NanoImprint Lithography resist. Microelectron. Eng. 2011, 88, 2096-2099. [CrossRef] 
26. DePalma, V.; Tillman, N. Friction and Wear of Self-Assembled Trichlorosilane Monolayer Films on Silicon. Langmuir 1989, 5, 868-872. [CrossRef]

27. Haensch, C.; Hoeppener, S.; Schubert, U.S. Chemical modification of self-assembled silane based monolayers by surface reactions. Chem. Soc. Rev. 2010, 39, 2323-2334. [CrossRef] [PubMed]

28. Rosales-Guzmán, C.; Forbes, A. How to Shape Light with Spatial Light Modulators; SPIE Press: Bellingham, DC, USA, 2017; ISBN 9781510613027.

29. Padgett, M.; Courtial, J.; Allen, L. Light's Orbital Angular Momentum. Phys. Today 2004, 57, 35-40. [CrossRef]

30. Jahani, S.; Jacob, Z. All-dielectric metamaterials. Nat. Nanotechnol. 2016, 11, 23-36. [CrossRef] [PubMed]

31. Staude, I.; Schilling, J. Metamaterial-inspired silicon nanophotonics. Nat. Photonics 2017, 11, $274-284$. [CrossRef]

32. Vaity, P.; Rusch, L. Perfect vortex beam: Fourier transformation of a Bessel beam. Opt. Lett. 2015, 40, 597-600. [CrossRef] [PubMed]

33. Goodman, J.W. Introduction to Fourier Optics; McGraw-Hill: New York, NY, USA, 1996; ISBN 9780070242548.

34. Gradshteyn, I.S.; Ryzhik, I.M. Table of Integrals, Series, and Products Eighth Edition; Elsevier Academic Press: Waltham, MA, USA, 2015; ISBN 9780123849335.

(C) 2018 by the authors. Licensee MDPI, Basel, Switzerland. This article is an open access article distributed under the terms and conditions of the Creative Commons Attribution (CC BY) license (http:/ / creativecommons.org/licenses/by/4.0/). 